\title{
Coping Styles and Sources of Stress of Undergraduate Health Science Students: An Integrative Review
}

\author{
*Robyn Marcella Henderson**, B. OT (UKZN). https://orcid.org/0000-0003-0806-654 I \\ Community service occupational therapist, Appelsbosch Hospital, KwaZulu-Natal, Durban, South Africa.
}

\author{
Odell Chetty**, B. OT (UKZN). https://orcid.org/0000-0002-2594-3 I 66
}

Community service occupational therapist, Addington Hospital, KwaZulu-Natal, Durban, South Africa.

\section{Thavanesi Gurayah. B. OT (UDW); M. OT (UDW). https://orcid.org/0000-000 I-9005-6355 \\ Lecturer, Occupational Therapy Department, School of Health Sciences, University of KwaZulu-Natal, Westville Campus, KwaZulu- Natal, Durban, South Africa.}

\section{**Undergraduate students at the time of the study}

Background and aim: Tertiary education provides many personal and social benefits. However, students, particularly those from professional and health science disciplines are known to face significantly greater stress throughout their undergraduate degrees. In this integrative review sources of stress for students and the strategies they use to cope with the stress are explored.

Method: Various databases were searched using common key phrases such as "stressors among undergraduate health science students" and "coping mechanisms utilised by undergraduate health science students". Thirty-three articles were selected based on predetermined inclusion and exclusion criteria. The content in the articles were analysed to identify themes.

Results: Academic stress was the primary source of stress for health science students. Other sources of stress included interpersonal interactions and clinical training. Students primarily used problem-focused strategies to cope with stress. Other strategies included avoidance and the use of social support networks.

Conclusion: Health science students experience stress from academic tasks, interpersonal stress and stresses emanating from clinical training. They utilize problem-focused coping strategies, as well as avoidance and seeking social support when faced with stressful situations. Students would benefit from regular screening for stress-related conditions, and interventions to teach them more adaptive ways to deal with stress.

Keywords: stressors, coping strategies, health science students, workload, student support.

\section{INTRODUCTION}

Higher education is essential for both personal success and economic growth. Tertiary education provides social benefits such as the acquisition of advanced knowledge, increased employability and financial stability'. However, successful graduation is a prerequisite for these benefits. Challenges to academic success include the complexity, number and timing of assessment tasks, and stress associated with intense studying at a higher education institution

Fears concerning the future, leaving behind friends and family, and having a poor work-life balance all take a toll on the mental health of university students ${ }^{2}$. This was confirmed by Makoni ${ }^{3}$, who stated that mental health remains a global concern for university students, as psychological distress impacts negatively on academic performance. Stressful factors experienced by students include rigid course structures, insufficient support systems, inadequate finances and conflicting role demands, due to either childrearing, caring for an elderly family member or part-time employment ${ }^{2}$. Moreover, many students are academically unprepared for higher education, as many are first generation students, who may lack the social capital to succeed ${ }^{4}$. This is reflected in their poor academic performance such as high failure and dropout rates, and lengthy throughput among others ${ }^{5}$.

This article is based on a review that was conducted by UKZN undergraduate students for the fulfilment of a bachelor's degree in Occupational Therapy. This review was underpinned by the following questions:

I. What are the sources of stress among undergraduate health science students?

2. What strategies do students use to cope with stress?

\section{METHOD}

An integrative review of the literature was conducted to understand the sources of stress, as well as the coping mechanisms utilised by undergraduate health science students. This method allowed for the inclusion of different types of studies for a holistic understanding of the research phenomenon ${ }^{6}$. This review was granted exemption from ethics, based on the pandemic, by the University of KwaZulu-Natal. The protocol reference number 00005806 was issued in response to the protocol submitted on 3I March 2020. 
Inclusion criteria:

- Articles published between 2000-2020

- Articles on undergraduate health science students.

- All articles were published in English and had to be full text.

Exclusion criteria:

- Articles involving medical students were excluded from the review as they are exposed to different stressors, and the demands and duration of their degree was different to that of other health science disciplines.

- Articles on postgraduate health science students were excluded as they may be exposed to different stressors, and cope differently.

\section{Review process}

The review process was done in phases. The following health databases were searched: Microsoft academic, Refseek, Catalogue search, SagePub, SciELO, Semantic Scholar, Science Direct, Google Scholar, Pubmed, Ebscohost and Medline were used. Pubmed and Medline were searched separately. The following descriptors and Boolean phrases were used as key phrases:

- undergraduate health science students,

- undergraduate occupational therapy students,

- coping mechanisms utilised by undergraduate health science students, OR undergraduate occupational therapy students,

- $\quad$ stressors among undergraduate health science students, OR undergraduate occupational therapy students

- AND sources of stress in undergraduate health science students OR undergraduate occupational therapy students.

The initial searches yielded 60 studies, and the title and abstracts were read in July 2020. The final sample consisted of 33 articles that met the inclusion criteria. The information to be extracted from the studies were defined and categorized, and the full-text articles were read. Data analysis entailed creating a table and extracting the information from all articles into sources of stress and coping strategies. Information was further broken down into themes such as academic, personal, environmental factors and other stressors, as well as adaptive and maladaptive coping strategies. Once all the information was extracted, the themes were constituted, reviewed, and refined, and a flow chart created. The review process is illustrated in Figure I (below).

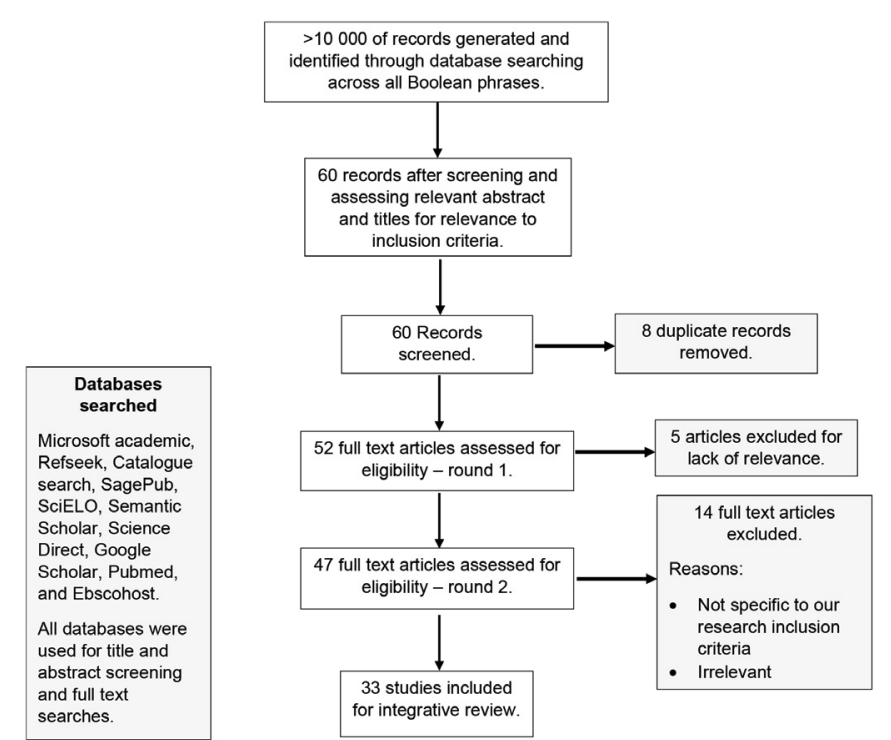

Figure I: Flow chart showing the research process.

\section{RESULTS}

Twenty five of the 33 studies were quantitative, six were qualitative, and two were systemic reviews. The studies were conducted in various countries including South Africa, Nigeria, Saudi Arabia, the United Kingdom, the United States of America, and Australia. The studies were focused on the perspectives of occupational therapy, physiotherapy, dental therapy, speech pathology, pharmacy, and nursing students. The articles included in this review are presented in Table I (p3 I), which are organized according to the numerical order of references.

The findings are presented according to the sources of stress and coping strategies utilized by students.

\section{Sources of stress}

Three major sources of stress were identified, which included academic activities, interpersonal/social interactions, and clinical training. Several studies identified academic demands as the most significant source of stress for health science students ${ }^{7-9}$. Students described examinations and a high workload as the major factors contributing to academic stress ${ }^{10-12}$. Interpersonal or social stressors with family and peers were cited in 15 studies $^{7,11,13-25}$. In another study conducted in Saudi Arabia, nursing students identified relationships with hospital staff to be a source of stress ${ }^{26}$.

Stress from clinical training was emphasised by 13 out of the 33 studies $^{7,13,17,18,20,25-32}$. Poorly staffed clinical departments, fast-paced wards and initial clinical placements were all factors contributing towards the stress of students ${ }^{28}$. High level patient care was also a prevalent stress factor amongst nursing students ${ }^{13,17,20}$. Other stressors that were reported less frequently included personal issues, financial concerns, environmental factors, psychosocial issues, and lack of students' professional skills. Personal stressors consisted primarily of students being stressed about their personal health-related conditions and illnesses ${ }^{7}$.

Financial issues were cited in a study by Gibbons, Dempster and Moutray ${ }^{28}$, where students revealed that they had to work part-time during their undergraduate studies to support themselves. Environmental factors cited as stress inducing included noisy living environments ${ }^{13}$. In terms of psychosocial stressors, final year physiotherapy students described the suffering and death of their patients as a traumatic experience ${ }^{39}$. Another study revealed that high parental expectations and decreased time to participate in leisure activities contributed to the students' psychosocial stress ${ }^{10}$.

Lack of professional knowledge was reported as a source of stress in six out of the 33 studies $^{13,17,20,23,25,27}$. Students felt that they lacked the necessary skills, and that clinical training was not always sufficient ${ }^{40}$. Other stressors identified in this review were concerns about postgraduate opportunities and career choices ${ }^{7}$. The frequency with which the stressors were identified in the reviewed studies is illustrated in Figure 2.

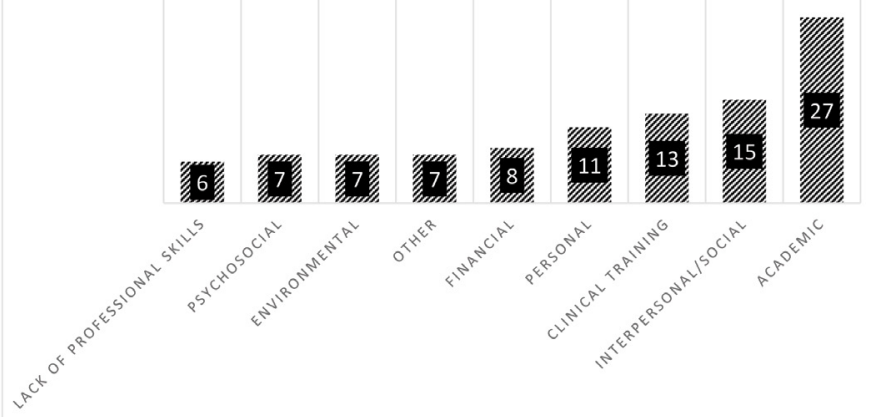

Figure 2: Sources of stress amongst health science students. 
Table I: Description of studies showing title, year, methodology, authors, country and findings of study.

\begin{tabular}{|c|c|c|c|c|c|c|}
\hline $\begin{array}{l}\text { Title of the journal } \\
\text { article }\end{array}$ & Authors & Year & Sample size & Methodology & Findings & Country \\
\hline $\begin{array}{l}\text { Sources of stress in a phar- } \\
\text { macy student population }{ }^{7}\end{array}$ & $\begin{array}{l}\text { Garber, M., Huston, } \\
\text { S., \& Breese, C. }\end{array}$ & 2019 & 721 & $\begin{array}{l}\text { Quantitative: survey } \\
\text { using the Perceived } \\
\text { Stress Scale. }\end{array}$ & $\begin{array}{l}\text { Stressors included aca- } \\
\text { demic work, pressure to } \\
\text { succeed, relationships, } \\
\text { postgraduate opportuni- } \\
\text { ties, and financial stress. }\end{array}$ & United States \\
\hline $\begin{array}{l}\text { Perceived stress and } \\
\text { sources of stress among } \\
\text { physiotherapy students } \\
\text { from three countries }{ }^{8}\end{array}$ & $\begin{array}{l}\text { Jacobs, T., } \\
\text { Gummesson, } \\
\text { C., Nordmark, } \\
\text { E., Ansary, D., } \\
\text { Remedios, L., \& } \\
\text { Webb, G. }\end{array}$ & 2018 & 626 & $\begin{array}{l}\text { Quantitative: cross- } \\
\text { sectional study } \\
\text { design, using the } \\
\text { Perceived Stress } \\
\text { Scale (PSS), and } \\
\text { the Undergraduate } \\
\text { Source of Stress } \\
\text { Questionnaire } \\
\text { (USOS). }\end{array}$ & $\begin{array}{l}\text { Academic factors were } \\
\text { found to be the most } \\
\text { significant, and financial } \\
\text { stressors were least } \\
\text { significant. }\end{array}$ & $\begin{array}{l}\text { Israel, } \\
\text { Australia, and } \\
\text { Sweden }\end{array}$ \\
\hline $\begin{array}{l}\text { Sources of stress and } \\
\text { psychological morbid- } \\
\text { ity among undergraduate } \\
\text { physiotherapy students }\end{array}$ & $\begin{array}{l}\text { Walsh, J., Feeney, C., } \\
\text { Hussey, J., \& Don- } \\
\text { nellan, C. }\end{array}$ & 2010 & 125 & $\begin{array}{l}\text { Quantitative: } \\
\text { survey using the } \\
\text { Undergraduate } \\
\text { Sources of Stress } \\
\text { Questionnaire and } \\
\text { the General Health } \\
\text { Questionnaire }\end{array}$ & $\begin{array}{l}\text { Students experienced } \\
\text { academic and personal } \\
\text { stressors. } 27 \% \text { of the } \\
\text { students presented with } \\
\text { psychological morbidity. }\end{array}$ & Ireland \\
\hline $\begin{array}{l}\text { A cross-sectional study } \\
\text { of stress and its sources } \\
\text { among health professional } \\
\text { students at Makerere } \\
\text { University, Uganda }{ }^{10}\end{array}$ & $\begin{array}{l}\text { Amanya, S. B., } \\
\text { Nakitende, J., \& } \\
\text { Ngabirano, T. D. }\end{array}$ & 2017 & 258 & $\begin{array}{l}\text { Quantitative: } \\
\text { descriptive cross- } \\
\text { sectional study } \\
\text { design, using the } \\
\text { General Health } \\
\text { Questionnaire } \\
\text { (GHQ-12). }\end{array}$ & $\begin{array}{l}\text { Stressors included the } \\
\text { academic curriculum } \\
\text { ( } 38 \%) \text {, high parental } \\
\text { expectations }(26.7 \%) \\
\text { and financial problems } \\
(24.4 \%) .\end{array}$ & Uganda \\
\hline $\begin{array}{l}\text { Nature of Stress among } \\
\text { Health Science Students in } \\
\text { a Malaysian University"l }\end{array}$ & $\begin{array}{l}\text { Othman, C., Fa- } \\
\text { rooqui, M., Yusoff, } \\
\text { M., \& Adawiyah, R. }\end{array}$ & 2013 & 248 & $\begin{array}{l}\text { Quantitative: } \\
\text { cross-sectional } \\
\text { study, using the } \\
\text { Medical Student } \\
\text { Stress Question- } \\
\text { naire }\end{array}$ & $\begin{array}{l}\text { Female students }(60 \%) \\
\text { were more stressed com- } \\
\text { pared to male students. }\end{array}$ & Malaysia \\
\hline $\begin{array}{l}\text { Source of stressors and } \\
\text { emotional disturbances } \\
\text { among undergraduate sci- } \\
\text { ence students in Malaysia }\end{array}$ & $\begin{array}{l}\text { Radeef, A.S., Faisal, } \\
\text { G.G., Ali, S.M., \& } \\
\text { Ismail, M.K. }\end{array}$ & 2014 & 194 & $\begin{array}{l}\text { Quantitative: } \\
\text { Cross sectional, } \\
\text { questionnaire-using } \\
\text { the Depression } \\
\text { Anxiety Stress Scale } \\
\text { (DASS-2I). }\end{array}$ & $\begin{array}{l}\text { Academic stress was } \\
\text { most significant, followed } \\
\text { by social /interpersonal } \\
\text { stressors. }\end{array}$ & Malaysia \\
\hline $\begin{array}{l}\text { Assessment of Level and } \\
\text { Sources of Stress Among } \\
\text { Allied Health Sciences } \\
\text { Students of Bayero Univer- } \\
\text { sity Kano: A Comparison } \\
\text { Between Clinical and Pre- } \\
\text { Clinical Students }^{13}\end{array}$ & $\begin{array}{l}\text { Muhammad, D., } \\
\text { Ahmad, A., \& } \\
\text { Usman, J. }\end{array}$ & 2019 & 230 & $\begin{array}{l}\text { Quantitative: } \\
\text { Using the MSSQ } \\
\text { (Medical Student } \\
\text { Stressors } \\
\text { Questionnaire). }\end{array}$ & $\begin{array}{l}\text { Academic stress was } \\
\text { prevalent, with anxiety } \\
(84.5 \%) \text {, depression } \\
(64.4 \%) \text {, and stress } \\
(56.7 \%) \text { rates recorded. }\end{array}$ & Nigeria \\
\hline $\begin{array}{l}\text { OT students' experience } \\
\text { of stress and coping }{ }^{14}\end{array}$ & $\begin{array}{l}\text { Govender, P., } \\
\text { Mkhabela, S., } \\
\text { Hlongwane, M., } \\
\text { Jalim, K., \& Jetha, } \\
\text { C. G }\end{array}$ & 2015 & 99 & $\begin{array}{l}\text { Quantitative: } \\
\text { descriptive survey } \\
\text { design using a de- } \\
\text { scriptive stress sur- } \\
\text { vey and the Ways } \\
\text { of Coping Checklist } \\
\text { (WCC). }\end{array}$ & $\begin{array}{l}\text { Academic stress was } \\
\text { most prevalent, and } \\
7.8 \% \text { of students were } \\
\text { mildly stressed, } 49.1 \% \\
\text { were moderately } \\
\text { stressed, } 6 \% \text { were highly } \\
\text { stressed, and } 0.4 \% \\
\text { were severely stressed. }\end{array}$ & South Africa \\
\hline
\end{tabular}




\begin{tabular}{|c|c|c|c|c|c|c|}
\hline $\begin{array}{l}\text { Title of the journal } \\
\text { article }\end{array}$ & Authors & Year & Sample size & Methodology & Findings & Country \\
\hline $\begin{array}{l}\text { Resilience in occupational } \\
\text { therapy students }{ }^{15}\end{array}$ & $\begin{array}{l}\text { De Witt, P.A., } \\
\text { Monareng, L., } \\
\text { Abraham, A.A.H., } \\
\text { Koor, S., \& Saber, R. }\end{array}$ & 2019 & 118 & $\begin{array}{l}\text { Quantitative: } \\
\text { Descriptive, cross- } \\
\text { sectional survey } \\
\text { design using the Re- } \\
\text { siliency Scale (RS); } \\
\text { the Perceived Stress } \\
\text { Visual Analogue } \\
\text { Scale (PSS) and the } \\
\text { Health Behaviours } \\
\text { Questionnaire } \\
\text { (HBQ). }\end{array}$ & $\begin{array}{l}\text { Academic stressors were } \\
\text { the most prevalent, as } \\
\text { well as social and per- } \\
\text { sonal stressors }\end{array}$ & South Africa \\
\hline $\begin{array}{l}\text { Replacing stressful chal- } \\
\text { lenges with positive coping } \\
\text { strategies: a resilience pro- } \\
\text { gram for clinical placement } \\
\text { learning }{ }^{16}\end{array}$ & $\begin{array}{l}\text { Delany, C., Miller, K. } \\
\text { J., El-Ansary, D., Re- } \\
\text { medios, L., Hosseini, } \\
\text { A., \& McLeod, S. }\end{array}$ & 2015 & 6 & $\begin{array}{l}\text { Qualitative: } \\
\text { A resilience pro- } \\
\text { gram was designed } \\
\text { consisting of four } \\
90 \text {-minute sessions. }\end{array}$ & $\begin{array}{l}\text { Clinical training was } \\
\text { stressful, and students } \\
\text { used avoidant coping. }\end{array}$ & Australia \\
\hline $\begin{array}{l}\text { Nursing students' } \\
\text { perceived stress, coping } \\
\text { strategies, health, and } \\
\text { supervisory approaches in } \\
\text { clinical practice: A Slovak } \\
\text { and Czech perspective }{ }^{17}\end{array}$ & $\begin{array}{l}\text { Gurková, E., \& } \\
\text { Zeleníková, R. }\end{array}$ & 2018 & 275 & $\begin{array}{l}\text { Quantitative: } \\
\text { cross-sectional, } \\
\text { descriptive study } \\
\text { using the Perceived } \\
\text { Stress Scale and the } \\
\text { Coping Behaviour } \\
\text { Inventory. }\end{array}$ & $\begin{array}{l}\text { Stressors categorized } \\
\text { as clinical, academic } \\
\text { and social stressors. } \\
\text { Problem focused coping, } \\
\text { avoidance, transference } \\
\text { and remaining positive } \\
\text { were used. }\end{array}$ & $\begin{array}{l}\text { Czech } \\
\text { Republic and } \\
\text { Slovakia }\end{array}$ \\
\hline $\begin{array}{l}\text { Stress and coping styles in } \\
\text { Japanese Nursing stu- } \\
\text { dents }^{18}\end{array}$ & $\begin{array}{l}\text { Yamashita, K., Saito, } \\
\text { M, \& Takao T }\end{array}$ & 2012 & 1324 & $\begin{array}{l}\text { Quantitative: } \\
\text { using the General } \\
\text { Health Question- } \\
\text { naire and Brief } \\
\text { Coping Orienta- } \\
\text { tions to Problems } \\
\text { Experienced scales. }\end{array}$ & $\begin{array}{l}\text { Academic stressors, } \\
\text { interpersonal } \\
\text { relationships, and stress } \\
\text { from clinical practice. } \\
\text { Strategies included social } \\
\text { support (61.7\%) and } \\
\text { avoidant coping ( } 2.7 \%) \text {. }\end{array}$ & Japan \\
\hline $\begin{array}{l}\text { Study of stress level in } \\
\text { occupational therapy } \\
\text { students during their aca- } \\
\text { demic curriculum } 19\end{array}$ & $\begin{array}{l}\text { Kumar, S., \& Jejurka, } \\
\text { K. }\end{array}$ & 2005 & 100 & $\begin{array}{l}\text { Quantitative: } \\
\text { study using a } \\
\text { 28-item stress } \\
\text { questionnaire and } \\
\text { Zung's self-rating } \\
\text { scale for depres- } \\
\text { sion. }\end{array}$ & $\begin{array}{l}\text { First year students } \\
\text { experienced greater } \\
\text { academic, social and } \\
\text { emotional stress. }\end{array}$ & India \\
\hline $\begin{array}{l}\text { A literature review on } \\
\text { stress and coping strategies } \\
\text { in Nursing students }{ }^{20}\end{array}$ & Leodoro J & 2017 & 13 & Systemic review & $\begin{array}{l}\text { Stressors included } \\
\text { the care of patients, } \\
\text { academics, and negative } \\
\text { interactions with staff. } \\
\text { Problem solving strategies } \\
\text { were used. }\end{array}$ & Saudi Arabia \\
\hline $\begin{array}{l}\text { Alcohol use by } \\
\text { occupational therapy } \\
\text { students: An exploratory } \\
\text { study }{ }^{2 !}\end{array}$ & $\begin{array}{l}\text { McCombie, RP., Ev- } \\
\text { ans, A., \& Miller, MJ }\end{array}$ & 2016 & 81 & $\begin{array}{l}\text { Quantitative: } \\
\text { using the Modified } \\
\text { version of the } \\
\text { Michigan alcohol } \\
\text { screening test, } \\
\text { the Stecker Life } \\
\text { Stressors Survey }\end{array}$ & $\begin{array}{l}\text { Alcohol use was } \\
\text { associated with } \\
\text { socialisation. Social } \\
\text { support was the primary } \\
\text { coping mechanism. }\end{array}$ & United States \\
\hline $\begin{array}{l}\text { Psychological distress } \\
\text { among nursing, } \\
\text { physiotherapy and } \\
\text { occupational therapy } \\
\text { students: A longitudinal } \\
\text { and predictive study }\end{array}$ & $\begin{array}{l}\text { Nerdrum, P., \& } \\
\text { Rustoen, T. }\end{array}$ & 2009 & 416 & $\begin{array}{l}\text { Quantitative: } \\
\text { using the StudData } \\
\text { questionnaire and } \\
\text { the General Health } \\
\text { Questionnaire }\end{array}$ & $\begin{array}{l}\text { Students experienced } \\
\text { academic stress and had } \\
\text { interpersonal problems. }\end{array}$ & Norway \\
\hline $\begin{array}{l}\text { Psychosocial stress factors } \\
\text { among mental health nurs- } \\
\text { ing students in KSA }{ }^{23}\end{array}$ & Seham, M & 2019 & 10 & $\begin{array}{l}\text { Qualitative: Inter- } \\
\text { pretive qualitative } \\
\text { approach involving } \\
\text { semi-structured } \\
\text { interviews }\end{array}$ & $\begin{array}{l}\text { Stressors included the } \\
\text { academic environment, } \\
\text { social distress, financial } \\
\text { constraints, and interper- } \\
\text { sonal relationships. }\end{array}$ & Saudi Arabia \\
\hline
\end{tabular}




\begin{tabular}{|c|c|c|c|c|c|c|}
\hline $\begin{array}{l}\text { Title of the journal } \\
\text { article }\end{array}$ & Authors & Year & Sample size & Methodology & Findings & Country \\
\hline $\begin{array}{l}\text { Experienced stressors and } \\
\text { coping strategies among } \\
\text { Iranian nursing students }{ }^{24}\end{array}$ & $\begin{array}{l}\text { Seyedfatemi, N., } \\
\text { Tafreshi, M., \& } \\
\text { Hagani, H. }\end{array}$ & 2007 & 440 & $\begin{array}{l}\text { Quantitative: } \\
\text { descriptive cross- } \\
\text { sectional study } \\
\text { using the Student } \\
\text { Stress Survey and } \\
\text { the Adolescent } \\
\text { Coping Orientation } \\
\text { for Problems Expe- } \\
\text { rienced Inventory. }\end{array}$ & $\begin{array}{l}\text { Stressors included } \\
\text { academic and } \\
\text { environmental stress, } \\
\text { interpersonal problems, } \\
\text { and health concerns. }\end{array}$ & Iran \\
\hline $\begin{array}{l}\text { Stress and coping strate- } \\
\text { gies among nursing } \\
\text { students: an international } \\
\text { study }^{25}\end{array}$ & Labrague et al & 2018 & $\begin{array}{l}547 \text { ( } 16 \mathrm{I} \\
\text { Greek } \\
\text { students, } \\
\text { I } 53 \text { Filipino } \\
\text { students, and } \\
233 \text { Nigerian } \\
\text { students) }\end{array}$ & $\begin{array}{l}\text { Quantitative: } \\
\text { Using The Per- } \\
\text { ceived Stress Scale } \\
\text { (PSS) and the } \\
\text { Coping Behaviour } \\
\text { Inventory }(\mathrm{CBI}) \text {. }\end{array}$ & $\begin{array}{l}\text { Academic stress was } \\
\text { ranked the highest } \\
(M=2.46, S D=0.78) \text {. } \\
\text { Problem focused coping } \\
\text { was the most used } \\
\text { coping strategy }(M=2.68 \text {, } \\
S D=0.72) \text {. }\end{array}$ & $\begin{array}{l}\text { Greece, } \\
\text { Philippines } \\
\text { and Nigeria }\end{array}$ \\
\hline $\begin{array}{l}\text { Examining stress percep- } \\
\text { tions and coping strate- } \\
\text { gies among Saudi Nursing } \\
\text { students } 26\end{array}$ & Labrague L.J. & 2018 & $\begin{array}{l}\text { I I quantita- } \\
\text { tive studies. }\end{array}$ & Systemic review & $\begin{array}{l}\text { Academic stress was the } \\
\text { most significant stressor. } \\
\text { Students utilized active } \\
\text { and passive coping styles. }\end{array}$ & Saudi Arabia \\
\hline $\begin{array}{l}\text { Hong Kong baccalaureate } \\
\text { nursing students' stress and } \\
\text { their coping strategies in } \\
\text { clinical practice } 27\end{array}$ & $\begin{array}{l}\text { Chan, C., So, W., \& } \\
\text { Fong, D }\end{array}$ & 2009 & 205 & $\begin{array}{l}\text { Quantitative: } \\
\text { survey using the } \\
\text { Perceived stress } \\
\text { scale, Physio-Psy- } \\
\text { cho-Social response } \\
\text { scale and Coping } \\
\text { Behaviour Inven- } \\
\text { tory. }\end{array}$ & $\begin{array}{l}\text { A lack of clinical } \\
\text { knowledge, taking care } \\
\text { of clients and academic } \\
\text { stressors induced } \\
\text { stress. Coping strategies } \\
\text { included problem solving, } \\
\text { transference, avoidance } \\
\text { and remaining positive. }\end{array}$ & Hong Kong \\
\hline $\begin{array}{l}\text { Stress and eustress in nurs- } \\
\text { ing students }{ }^{28}\end{array}$ & $\begin{array}{l}\text { Gibbons, C., Demp- } \\
\text { ster, M., \& Moutray, } \\
\text { M. }\end{array}$ & 2008 & 16 & $\begin{array}{l}\text { Qualitative: } \\
\text { focus group inter- } \\
\text { views }\end{array}$ & $\begin{array}{l}\text { Students who coped well } \\
\text { used support networks } \\
\text { and were positive, to- } \\
\text { wards programme issues }\end{array}$ & $\begin{array}{l}\text { United } \\
\text { Kingdom }\end{array}$ \\
\hline $\begin{array}{l}\text { Stress, coping and satisfac- } \\
\text { tion in nursing students }{ }^{29}\end{array}$ & $\begin{array}{l}\text { Gibbons, C., Demp- } \\
\text { ster, M., \& Moutray, } \\
\text { M. }\end{array}$ & 2011 & 171 & $\begin{array}{l}\text { Qualitative using } \\
\text { The General Health } \\
\text { Questionnaire. }\end{array}$ & $\begin{array}{l}\text { Stressors included } \\
\text { academic and fieldwork } \\
\text { demands. Coping strate- } \\
\text { gies included avoidance } \\
\text { and using social support. }\end{array}$ & $\begin{array}{l}\text { United } \\
\text { Kingdom }\end{array}$ \\
\hline $\begin{array}{l}\text { Perceived stressors of oral } \\
\text { hygiene students in the } \\
\text { dental environment }{ }^{30}\end{array}$ & $\begin{array}{l}\text { Gordon, N., Rayner, } \\
\text { C., Wilson, V., } \\
\text { Crombie, K., Shaikh, } \\
\text { A., \& Yasin-Harnek- } \\
\text { ar, S }\end{array}$ & 2016 & $\begin{array}{l}89 \text { ( } 3^{\text {rd }} \text { year } \\
\text { Bachelor of } \\
\text { Oral Health } \\
\text { students) }\end{array}$ & $\begin{array}{l}\text { Quantitative: } \\
\text { Using the DES } \\
\text { questionnaire; } \\
\text { and the Maslach } \\
\text { Burnout Inventory } \\
\text { (MBI). }\end{array}$ & $\begin{array}{l}\text { First and second year } \\
\text { students identified theo- } \\
\text { retical learning as most } \\
\text { stressful, whereas the } \\
\text { 3rd year group reported } \\
\text { clinical training as most } \\
\text { stressful. }\end{array}$ & South Africa \\
\hline $\begin{array}{l}\text { Perceived stress and well- } \\
\text { being amongst Dental Hy- } \\
\text { giene and Dental Therapy } \\
\text { Students }\end{array}$ & $\begin{array}{l}\text { Harris, M., Wilson, } \\
\text { J., Holmes, S., \& } \\
\text { Radford, D. }\end{array}$ & 2017 & $\begin{array}{l}72 \text { dental } \\
\text { hygiene and } \\
\text { dental thera- } \\
\text { py students, } \\
\text { and } 80 \text { final } \\
\text { year out- } \\
\text { reach dental } \\
\text { students (as } \\
\text { a comparison } \\
\text { group) }\end{array}$ & $\begin{array}{l}\text { Quantitative: } \\
\text { survey using the } \\
\text { Dental Environment } \\
\text { Stress questionnaire } \\
\text { (DES); Depres- } \\
\text { sion Anxiety Stress } \\
\text { Scales (DASS2I); } \\
\text { Scales of Psycho- } \\
\text { logical Well-Being } \\
\text { (SPWB); Valuing } \\
\text { Questionnaire } \\
\text { (VQ); and the Adult } \\
\text { Hope Scale (AHS). }\end{array}$ & $\begin{array}{l}\text { Stressors included } \\
\text { academic work and field- } \\
\text { work practice. }\end{array}$ & $\begin{array}{l}\text { United } \\
\text { Kingdom }\end{array}$ \\
\hline
\end{tabular}




\begin{tabular}{|c|c|c|c|c|c|c|}
\hline $\begin{array}{l}\text { Title of the journal } \\
\text { article }\end{array}$ & Authors & Year & Sample size & Methodology & Findings & Country \\
\hline $\begin{array}{l}\text { Stress sources in nursing } \\
\text { practice. Evolution during } \\
\text { nursing training } \\
32\end{array}$ & $\begin{array}{l}\text { Zupiria Gorostidi, } \\
\text { X., Huitzi Egilegor, } \\
\text { X., Jose Alberdi } \\
\text { Erice, M., Jose } \\
\text { Uranga Iturriotz, M., } \\
\text { Eizmendi Garate, I., } \\
\text { Barandiaran Lasa, } \\
\text { M., \& Sanz Cas- } \\
\text { cante, X. }\end{array}$ & 2007 & 69 & $\begin{array}{l}\text { Quantitative: } \\
\text { Using components } \\
\text { of the KEZKAK, } \\
\text { STAI and demo- } \\
\text { graphic question- } \\
\text { naires. }\end{array}$ & $\begin{array}{l}\text { Stressors included } \\
\text { academic and social } \\
\text { stressors, and the lack of } \\
\text { professional knowledge. }\end{array}$ & Spain \\
\hline $\begin{array}{l}\text { Perceptions of stress, time } \\
\text { management and coping } \\
\text { strategies of speech pa- } \\
\text { thology students on clinical } \\
\text { placement }^{33}\end{array}$ & $\begin{array}{l}\text { Lincoln, M., \& } \\
\text { Adamson, B. }\end{array}$ & 2004 & $\begin{array}{l}\text { Time l: } 62 \\
\text { Time 2: } 36 \\
\text { Time 3: } 48\end{array}$ & $\begin{array}{l}\text { Quantitative: } \\
\text { Using the Perceived } \\
\text { Stress Scale and } \\
\text { Australian Time } \\
\text { and Organization } \\
\text { Management Scale } \\
\text { (ATOMS). }\end{array}$ & $\begin{array}{l}\text { Coping strategies in- } \\
\text { cluded problem focused } \\
\text { coping, optimism and } \\
\text { using social support. }\end{array}$ & Australia \\
\hline $\begin{array}{l}\text { Causes of Stress and Cop- } \\
\text { ing Strategies Adopted } \\
\text { by Undergraduate Health } \\
\text { Professions Students in a } \\
\text { University in the United } \\
\text { Arab Emirates }{ }^{34}\end{array}$ & $\begin{array}{l}\text { Gomathi, K., } \\
\text { Ahmed, S., \& Sreed- } \\
\text { haran, J. }\end{array}$ & 2013 & 212 & $\begin{array}{l}\text { Quantitative: } \\
\text { survey using the } \\
\text { brief COPE inven- } \\
\text { tory }\end{array}$ & $\begin{array}{l}\text { Stressors included } \\
\text { academic and health } \\
\text { related stress. Religion } \\
\text { and praying were coping } \\
\text { strategies. }\end{array}$ & $\begin{array}{l}\text { United Arab } \\
\text { Emirates }\end{array}$ \\
\hline $\begin{array}{l}\text { The role of religiosity, cop- } \\
\text { ing strategies, self-efficacy } \\
\text { and personality dimensions } \\
\text { in the prediction of Iranian } \\
\text { undergraduate rehabilita- } \\
\text { tion interns' satisfaction } \\
\text { with their clinical experi- } \\
\text { ence }^{35}\end{array}$ & $\begin{array}{l}\text { Mirsaleh, Y., Rezai, } \\
\text { H., Kivi, S., \& } \\
\text { Ghorbani, R. }\end{array}$ & 2010 & 318 & $\begin{array}{l}\text { Quantitative: } \\
\text { cross-sectional } \\
\text { survey using the } \\
\text { Islamic Religios- } \\
\text { ity Scale, Ways of } \\
\text { Coping Question- } \\
\text { naire, General } \\
\text { Self-efficacy Scale, } \\
\text { NEO Five Fac- } \\
\text { tor Inventory, and } \\
\text { Satisfaction with } \\
\text { Clinical Experiences } \\
\text { Questionnaire. }\end{array}$ & $\begin{array}{l}\text { Religiosity, problem-fo- } \\
\text { cused coping and general } \\
\text { self-efficacy were good } \\
\text { predictors of satisfaction } \\
\text { with clinical internship in } \\
\text { rehabilitation students. }\end{array}$ & Iran \\
\hline $\begin{array}{l}\text { Nursing students' stress } \\
\text { and coping strategies dur- } \\
\text { ing clinical training in } \mathrm{KSA}^{36}\end{array}$ & $\begin{array}{l}\text { Waled, A.M., \& } \\
\text { Badria, M.A. }\end{array}$ & 2019 & 125 & $\begin{array}{l}\text { Quantitative: } \\
\text { Descriptive cross- } \\
\text { sectional study }\end{array}$ & $\begin{array}{l}\text { Stressors included } \\
\text { academic stress, a lack of } \\
\text { professional knowledge } \\
\text { and competitive relation- } \\
\text { ships with peers. }\end{array}$ & Saudi Arabia \\
\hline $\begin{array}{l}\text { Dealing with stress: } \\
\text { Patterns of self-comfort } \\
\text { among healthcare stu- } \\
\text { dents }^{37}\end{array}$ & $\begin{array}{l}\text { Vera Dahlqvist, A. S., } \\
\text { \& Astrid, N. }\end{array}$ & 2018 & 168 & $\begin{array}{l}\text { Qualitative: } \\
\text { focus group. }\end{array}$ & $\begin{array}{l}\text { Emotion focused styles of } \\
\text { coping were used. }\end{array}$ & Sweden \\
\hline $\begin{array}{l}\text { Predictors of mental dis- } \\
\text { tress among undergradu- } \\
\text { ate health science students } \\
\text { of Hawassa University, } \\
\text { College of Medicine and } \\
\text { Health Sciences, Hawassa, } \\
\text { SNNPR, Ethiopia: a cross- } \\
\text { sectional study }\end{array}$ & $\begin{array}{l}\text { Bedaso, A., Duko, } \\
\text { B., \& Yeneabat, T. }\end{array}$ & 2020 & 309 & $\begin{array}{l}\text { Quantitative: } \\
\text { cross-sectional } \\
\text { study design }\end{array}$ & $\begin{array}{l}\text { A lack of social support } \\
\text { and the excessive use of } \\
\text { substances were strong } \\
\text { predictors of mental } \\
\text { distress. }\end{array}$ & Ethiopia \\
\hline
\end{tabular}

\section{Strategies used by students to cope with stress}

Students used a variety of strategies to help them cope with stress. Problem-focused coping was identified as one of the most used coping strategies ${ }^{14,20,25}$. Avoidance was another commonly used strategy, which involved students waiting for others to solve their problems, or avoiding teachers, but this approach led to difficulties in clinical training ${ }^{33}$. Other negative coping strategies that were not health promoting was avoidance (17\%), transference $(11 \%)$ and substance use by $2 \%$ of the sample.

Students used social support networks as a strategy for coping, as indicated in eight of the 33 studies $^{14,16,18,21,24,29,33,39}$. The frequency with which various coping strategies were used by students in the 


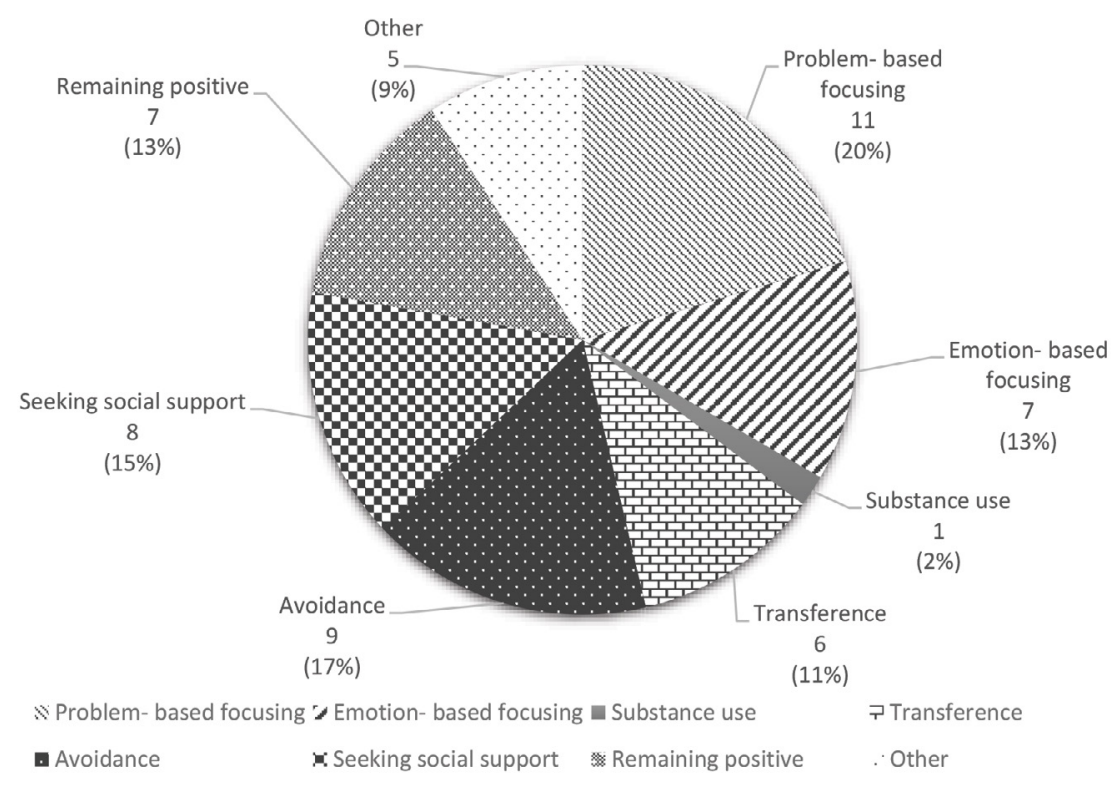

Figure 3: Pie graph showing coping strategies of students.

reviewed studies can be seen in Figure 3.

\section{DISCUSSION}

Health science students experience significantly higher levels of stress as compared to students in other disciplines ${ }^{41}$. Academic related stress was most prevalent amongst health science students ${ }^{7-9}$ and appeared to increase over the four-year occupational therapy course, with final year students perceiving it to be the greatest source of stress ${ }^{12}$. Examinations and an increased workload were most significant ${ }^{10-12}$, which was attributed to students' poor time management ${ }^{34}$. This was reiterated by Mohamadkhani et $a^{142}$ who stated that efficient time management skills were an important predictor for academic performance, reduced stress levels and increased productivity ${ }^{42}$.

Clinical training was also identified as a source of stress $^{28}$; due to understaffed clinical settings, high turnover in wards and problematic clinical placements. Garber et al. ${ }^{7}$ described fieldwork rotations to be stressful for pharmacy students, who were concerned about their performance in these experiential rotations. Negative relationships with faculty members were identified as one of the key social stressors ${ }^{7}$. A poor relationship with staff can result in decreased academic performance, as students find it difficult to approach lecturers or clinical supervisors. Social stress was cited in Gomathi et al. ${ }^{34}$, who reported that competitive relationships amongst peers was stressful. These competitive interpersonal relationships have been found to negatively impact on students' available support systems. Family conflicts were also found to compound the stress experienced by students, due to a negative impact on the students' available support networks ${ }^{21}$.

Gomathi et al. ${ }^{34}$, reported that the main psychosocial stressors experienced by students were high parental expectations, lack of adequate leisure time, and anxiety about the future, which centred about future unemployment. Psychosocial stresses of students included difficulties in processing the death or suffering of their patients ${ }^{39}$. Peters et $a l^{43}$, suggested that health providers are constantly exposed to the death and suffering of their clients, which can serve as a subconscious reminder of their own mortality. Students' health-related conditions were also stress inducing ${ }^{7,13}$. Students with chronic illnesses, or those who had surgeries found it difficult to manage these additional stressors ${ }^{7}$. Binge eating disor- ders and obesity were prevalent in first generation students ${ }^{44}$. These findings were in stark contrast to what has been reported in developing countries, such as South Africa, where many students presented with food insecurity and malnutrition ${ }^{45}$. DominguezWhitehead ${ }^{45}$ highlighted that South African university students were subjected to food insecurity because of limited funds.

Environmental factors such as noisy environments and the climate within the university and clinical settings were also stress inducing. Servilha and Delatti ${ }^{46}$, reported that increased noise levels were a major stressor for undergraduate health science students, which impacted their studies negatively. Financial stress was another source of stress identified within this integrative review. In one of the studies, it was found that students needed to secure part-time employment to alleviate their financial burdens ${ }^{28}$. University students with financial stress were likely to present with decreased academic performance, as they struggled to balance work and completing academic tasks ${ }^{47}$. This continues to be a problem particularly in developing countries, including South Africa, with many students on financial aid. A lack of professional knowledge was the least reported stressor within this integrative review. These students felt that clinical training was inadequate, and that they continued to lack the necessary clinical skills ${ }^{23}$.

\section{Coping strategies of health science students}

Students used a variety of coping strategies, including problem focused coping and emotion focused coping. It was found that students with high levels of resilience and optimism tended to respond to stress with adaptive coping strategies, such as task-oriented and active coping, whilst those with high levels of pessimism were likely to use maladaptive strategies, such as disengagement, denial, and venting of emotions. Some of the adaptive strategies included remaining positive and using social support. Other negative coping strategies included avoidance, transference, and substance use to cope with stress.

As can be seen in Figure 3, health science students were more likely to utilize problem-focused coping $(20 \%)^{14,17,20,24-27,34,35,36,41}$, in contrast to emotional-focused coping $(13 \%)^{14,16,24,34,35,37,39}$. Problem focused coping involves taking control of the stressor by either seeking information or assistance in handling the situation, whereas emotional focused coping included avoidance (I 7\%) ${ }^{17,24,25,27,29,33,34,35,36}$ and seeking social support ${ }^{14,16,18,21,24,29,33,35}$ (I5\%). Govender et al ${ }^{14}$ found that only $2 \%$ of occupational therapy students at the University of KwaZulu-Natal between the first and fourth year of study used problem-focused coping ${ }^{13}$. This strategy consisted of identifying, planning, analysing potential solutions and taking the necessary actions to solve the problem ${ }^{48}$. Other studies revealed that health science students employed a range of problem-focused coping strategies such as "managing their time, seeking information and sport and recreational activities, or they employ emotion-focused strategies such as tension reduction strategies, such as exercising, balanced diet, getting enough sleep, and engaging in constructive leisure activities"'|4.

\section{Adaptive coping strategies}

Remaining positive was cited in II out of the 33 studies included in this integrative review ${ }^{14,17,20,24-27,34,35,36,39}$. Students were found 
to adopt a positive attitude and mind-set in response to stressful events, confirming the use of hopefulness and optimism as a coping $s^{2} y \mathrm{e}^{36}$. A positive mind-set is likely to prevent students from resorting to helpless coping styles. Students were reported to be engaged in problem-focused methods of coping where they actively sought ways to cope with different stressors. Coping approaches included the development of solutions to their problems, and the seeking of assistance from student support services ${ }^{26}$. Another adaptive coping style identified was seeking social support. Support from family and friends is crucial in enabling a student to successfully integrate into university life, and a poor support network is a strong predictor of mental distress ${ }^{38}$. This approach entailed students talking about their problems with family and friends ${ }^{48}$. One study identified $61.7 \%$ of nursing students from a cohort of I 324 students who used social support as a means of coping ${ }^{17}$.

Emotion-focused coping was cited in seven out of the 33 articles $^{14,16,24,34,35,37,39}$, where the individual tried to minimise the stressor, and they felt better, but the problem remained unsolved. Other strategies used by students included remaining positive, transference, and other non-specific methods, such as remaining positive or optimistic, ${ }^{17,27}$ and substance use.

\section{Maladaptive coping strategies}

Students who engaged in avoidance behaviours often participated in activities such as procrastination, substance use and playing video games. These students were unlikely to identify the positive aspects of a situation ${ }^{24}$, which indicated a negative worldview and tendency to utilise avoidant coping styles. McCombie et $a^{21}$ postulated that some students used substance use as a coping strategy. Problematic family relationships resulted in a lack of social support, resulting in the increased tendency to use substances. With emotion-focused coping, the stressor is not analysed or resolved, but rather indirectly managed, and may include maladaptive coping strategies, such as substance use, avoidance, or transference, self-blame, denial, minimisation, sleeping, eating, wishful thinking and keeping to oneself.

\section{LIMITATIONS}

The limitation in this study was that the researchers were undergraduate students and novice researchers. This could have affected the analysis and information extracted during the integrative review. However, having the project supervised by a more experienced researcher mitigated this limitation.

\section{RECOMMENDATIONS}

Based on the researchers' analysis, the following recommendations are made:

- Health science and other professional degrees are associated with an increased academic workload, due to the clinical training component. Therefore, there is a need for health science disciplines to review their course structures, and the number and timing of assessments, to reduce the stress on students.

- Interventions to support students should be emphasised for first year orientation programmes. Thereafter student support services could send out electronic stress questionnaires to students, which could be conducted biannually, at the beginning and end of the academic year, allowing support services to screen for any students who may be struggling, and ensure timely support, nuanced to the students' needs ${ }^{39}$. Students who experienced the death of a patient can be referred to a psychologist or student counsellor to assist them in coping with this loss. Research has shown that students do experi- ence stress because of their patients' suffering and death ${ }^{49}$. Some believe that academic support should be compulsory for health science students, who are often reluctant to attend sessions voluntarily ${ }^{50}$. Moreover, it is recommended that student support services must address the academic and emotional needs of students, and support must be nuanced to the students' needs.

- Workshops aimed at addressing budgeting skills would be useful in assisting students to better manage their finances and reduce financial stress.

- The clinical supervisor-student bond may be strengthened during annual workshops for supervisors to ensure students are well supported during their fieldwork blocks. King, Edlington and Williams ${ }^{51}$, suggested that both the student and the clinical supervisor require training to ensure effective clinical supervision.

\section{CONCLUSION}

The purpose of this integrative review was to determine the sources of stress for health science students in undergraduate programmes, and strategies that they used to cope with stress as reported in international published peer reviewed articles. The sources of stress included academic demands, social and personal factors, financial worries, and stress as a result of clinical training. Other stressinducing factors included environmental influences, psychosocial factors, and feelings of inadequacy in relation to professional knowledge. Most students reported the use of problem-focused coping in response to increased stress, while others used maladaptive coping styles, such as substance use and other avoidant behaviours. The multitude of stressors and maladaptive coping necessitates the need for support to be timeously accessed by students. The implementation of online semester check-ins would be beneficial in allowing support services to identify students requiring additional support. Students' mental health are predictors for how well they will cope in tertiary education, and specifically with the demands of the health science programmes. It is imperative they access timely support, nuanced to their academic and personal needs.

\section{AUTHOR CONTRIBUTIONS}

Odell Chetty and Robyn Henderson conducted the initial article search, information extraction, data analysis, writing, editing and submission of the article. Thavanesi Gurayah conceptualised and supervised the project and edited the final manuscript.

\section{Acknowledgements}

The authors wish to acknowledge the other members of their research team Lerato Xulu, Nelly Zulu, Zama Mzobe and Nqobile Mhlope who assisted in conducting the review.

\section{REFERENCES:}

I. Chan R. Understanding the purpose of higher education: An analysis of the economic and social benefits for completing a college degree. Journal of Education Policy, Planning and Administration, 20 16; 6(5): I-40. https://www.researchgate.net/publication/305228497_ Understanding_the_purpose_of_higher_education_An_analysis_of_the_economic_and_social_benefits_for_completing_a_college_degree

2. Grabowski C, Rush M, Ragen K, Fayard V, Watkins-Lewis K. Today's Non-Traditional Student: Challenges to Academic Success and Degree Completion. Inquiries Journal/Student Pulse, 2016; 8(03).

3. Makoni M. Mental health a worry for student affairs worldwide. 
Journal of Student Affairs in Africa, 2016; 4(2): 65-66.

https://doi: 10.14426/jsaa.v4i2.1524

http://www.inquiriesjournal.com/a?id = 1377

4. Dhunpath R, Vithal R. Alternative access to higher education. Cape Town: Pearson. 2014. https://doi: I0.1 I63/22 I25868-1234009|

5. Tewari DD, llesanmi KD, Serpa S. Teaching and learning interaction in South Africa's higher education: Some weak links. Cogent Social Sciences, 2020; 6: I. https://doi: 10.1080/2331 I886.2020.1740519

6. Samaan, Z., Mbuagbaw, L., Kosa, D., Borg Debono, V., Dillenburg, R., Zhang, S., Fruci, V., Dennis, B., Bawor, M., \& Thabane, L. (20/3). A systematic scoping review of adherence to reporting guidelines in health care literature. Journal of multidisciplinary healthcare, 6, 169-188. https://doi.org/10.2147/JMDH.S43952

7. Garber M, Huston S, Breese C. Sources of stress in a pharmacy student population. Currents in Pharmacy Teaching and Learning, 2019; II (4): 329-337. https://doi:10.1016/j.cptl.2019.01.014

8. Jacobs T, Gummesson C, Nordmark E, Ansary D, Remedios L, Webb G. Perceived stress and sources of stress among physiotherapy students from 3 countries. Journal of Physical Therapy Education, 2012; 26(3): 57-65. https://doi:I0.1097/0000 I4 I6-201207000-00008

9. Walsh J, Feeney C, Hussey J, Donnellan C. Sources of stress and psychological morbidity among undergraduate physiotherapy students. Physiotherapy, 2010; 96(3): 206-212.

https://doi:10.1016/j.physio.2010.01.005

10. Amanya SB, Nakitende J, Ngabirano TD. A cross-sectional study of stress and its sources among health professional students at Makerere University, Uganda. Nursing open, 2017; 5(I): 70-76. https://doi.org/10.1002/nop2.113

II. Othman C, Farooqui M, Yusoff M, Adawiyah R. Nature of Stress among Health Science Students in a Malaysian University. Procedia - Social and Behavioral Sciences, 2013; 105: 249-257. https://doi:10.1016/j.sbspro.2013.11.026

12. Radeef AS, Faisal GG, Ali SM, Ismail MK. Source of stressors and emotional disturbances among undergraduate science students in Malaysia. International Journal of Medical Research \& Health Sciences, 20 14; 3(2). https://doi: 10.5958/j.2319-5886.3.2.082

13. Muhammad D, Ahmad A, Usman J. Assessment of Level and Sources of Stress among Allied Health Sciences Students of Bayero University Kano: A Comparison between Clinical and Pre-Clinical Students. Education in Medicine Journal, 2019; II(I): II-19.

https://doi:10.21315/eimj2019.1I.I.2

14. Govender P, Mkhabela S, Hlongwane M, Jalim K, Jetha CG. OT student's experience of stress and coping. South African Journal of Occupational Therapy, 2015; 45: 34-39.

http://dx.doi.org/10.17/59/2310-3833/2015/v45n3/a7

15. De Witt PA, Monareng L, Abraham AAH, Koor S, Saber R. Resilience in occupational therapy students. South African Journal of Occupational Therapy, 2019; 49(2): 33-4I.

https://dx.doi.org/I0.17159/23103833/2019/vol49n2a6

16. Delany C, Miller KJ, El-Ansary D, Remedios L, Hosseini A, McLeod S. Replacing stressful challenges with positive coping strategies: a resilience program for clinical placement learning. Advances in health sciences education: theory and practice, 20I5; 20(5): 1303-1324. https://doi.org/10.1007/s 10459-015-9603-3

17. Gurková E, Zeleníková R. Nursing students' perceived stress, coping strategies, health and supervisory approaches in clinical practice: $A$ Slovak and Czech perspective. Nursing Education Today, 2018; 65 : 4-10. https://doi.org/10.1016/j.nedt.2018.02.023

18. Yamashita K, Saito M, Takao T. Stress and coping styles in Japanese nursing students. International Journal of Nursing Practice. 2012; 18(5):489-496.
19. Kumar S, Jejurka K. Study of stress level in occupational therapy students during their academic curriculum. The Indian Journal of Occupational Therapy, 2005; 37(I): 5-14. http://dx.doi.org/10.17/59/2310-3833/2015/v45n3/a7

20. Leodoro J. A literature review on stress and coping strategies in nursing students. Journal of Mental Health, 2017; 25(4). https://do i: 10.1080/09638237.2016.1244721

2I. McCombie RP, Evans A, Miller MJ. Alcohol Use by Occupational Therapy Students: An Exploratory Study. Occupational Therapy in Mental Health, 2016; 32(I): I-15, https://doi:10.1080/0164212X.2015.1079514

22. Nerdrum P, Rustoen T. Psychological distress among nursing, physiotherapy and occupational therapy students: A longitudinal and predictive study. Scandinavian Journal of Educational Research, 2009; 53(4): 363-378. https://doi.org/10.1080/003 I3830903043। 33

23. Seham M. Psychosocial stress factors among mental health nursing students in KSA. Journal of Taibah University Medical Sciences, 2019; I4(I): 60-66. https://doi:10.1016/j.jtumed.2018.11.006

24. Seyedfatemi N, Tafreshi M, Hagani H. Experienced stressors and coping strategies among Iranian nursing students. BMC Nursing, 2007; 6(I). https://doi:I0.I I86/I472-6955-6-II

25. Labrague LJ, McEnroe-Petitte DM, Papathanasiou IV, Edet OB, Tsaras K, Leocadio MC, Colet P, Kleisiaris CF, Fradelos EC, Rosales RA, Vera Santos-Lucas K, Velacaria P. Stress and coping strategies among nursing students: an international study. Journal of Mental Health, 2018; 27(5): 402-408. https://doi.org/10.1080/09638237.2017.1417552

26. Labrague LJ, McEnroe-Petitte DM, De Los Santos J, Edet OB. Examining stress perceptions and coping strategies among Saudi nursing students: A systematic review. Nurse Education Today, 2018; 65: 192-200. https://doi.org/10.1016/j.nedt.2018.03.012

27. Chan C, So W, Fong D. Hong Kong baccalaureate nursing students' stress and their coping strategies in clinical practice. Journal of Professional Nursing, 2009; 25(5): 307-313.

https://doi:10.1016/j.profnurs.2009.01.018

28. Gibbons $C$, Dempster M, Moutray M. Stress and eustress in nursing students. Journal of Advanced Nursing, 2008; 6 I (3): 282-290. https://doi: I 0. I I I I/j. I365-2648.2007.04497.x

29. Gibbons C, Dempster M, Moutray M. Stress, coping and satisfaction in nursing students. Journal of Advanced Nursing, 201 I; 67(3): 62 I-632. https://doi.org/I0.1 I I I/j.1365-2648.2010.05495.x

30. Gordon N, Rayner C, Wilson V, Crombie K, Shaikh A, YasinHarnekar S. Perceived stressors of oral hygiene students in the dental environment. African Journal of Health Professions Education, 2016; 8(I): 20-24. https://doi:I0.7I96/AJHPE.20I6.v8il.422

31. Harris M, Wilson J, Holmes S, Radford D. Perceived stress and wellbeing among dental hygiene and dental therapy students. British Dental Journal, 2017; 222(2): 101-106. https://doi: 10.1038/sj.bdj.2017.76

32. Zupiria Gorostidi X, Huitzi Egilegor X, Jose Alberdi Erice M, Jose Uranga Iturriotz M, Eizmendi Garate I, Barandiaran Lasa M, Sanz Cascante $X$. Stress sources in nursing practice. Evolution during nursing training. Nurse Education Today, 2007; 27(7): 777-787. https://doi:10.1016/j.nedt.2006.10.017

33. Lincoln M, Adamson B. Perceptions of stress, time management and coping strategies of speech pathology students on clinical placement. Advances in speech language pathology. 2004; 6(2): 91-99. https://doi.org//0.1080/I44I70404100017085 I2

34. Gomathi K, Ahmed S, Sreedharan J. Causes of Stress and Coping Strategies Adopted by Undergraduate Health Professions Students in a University in the United Arab Emirates. Sultan Qaboos Univer- 
sity Medical Journal, 20I 3; 13(3): 430-434.

https://doi: I0.12816/0003267

35. Mirsaleh Y, Rezai H, Kivi S, Ghorbani R. The role of religiosity, coping strategies, self-efficacy, and personality dimensions in the prediction of Iranian undergraduate rehabilitation interns' satisfaction with their clinical experience. Clinical Rehabilitation, 20 10; 24(I2): I I36-I I 43. https://doi:10.1 I77/0269215510375907

36. Waled AM, Badria MA. Nursing students' stress and coping strategies during clinical training in KSA. Journal of Taibah University Medical Sciences, 2019; 14(2): 116-122.

https://doi.org/10.1016/j.jtumed.2019.02.002

37. Vera Dahlqvist AS, Astrid N. Dealing with stress: Patterns of selfcomfort among healthcare students. Nurse Education Today, 2018; 28(4): 476-84. https://doi:10.1016/j.nedt.2007.07.010.

38. Bedaso A, Duko B, Yeneabat T. Predictors of mental distress among undergraduate health science students of Hawassa University, College of Medicine and Health Sciences, Hawassa, SNNPR, Ethiopia: a cross-sectional study. Annals of general psychiatry, 2020; 19: 6. https://doi.org/I0.1 I86/s 1299I-020-0258-y

39. Van Vuuren E, Bodenstein K, Nel M. Stressors and coping strategies among physiotherapy students: Towards an integrated support structure. Health SA = SA Gesondheid, 2018; 23: 8-1091. https://doi.org/10.4102/hsag.v23i0.1091

40. Arzu Y, Emel B. The effect of mentoring program on adjustment to university and ways of coping with stress in nursing students: Aquasi-experimental study. Nurse Education Today, 2019; 80: 5258. https://doi.org/10.1016/j.nedt.2019.06.006

4I. Alkatheri A, Bustami R, Albekairy A, Alanizi A, Alnafesah R, Almodaimegh $\mathrm{H}$. Quality of Life and Stress Level among Health Professions Students. Health Professions Education, 2020; 6(2): 20I-2I 0. https://doi.org/10.1016/j.hpe.2019.11.004

42. Mohamadkhani Ghiasvand A, Naderi M, Zagheri Tafreshi M, Ahmadi F, Hosseini M. Relationship between time management skills and anxiety and academic motivation of nursing students in Tehran. Electronic physician, 2017; 9(1): 3678-3684.

43. Peters L, Cant R, Payne S, O'Connor M, McDermott F, Hood K. How Death Anxiety Impacts Nurses' Caring for Patients at the End of Life: A Review of Literature. The Open Nursing Journal, 2013; 7: |4-21. https://doi:10.2174/1874434601307010014

44. Lipson S, Sonneville K. Eating disorder symptoms among undergraduate and graduate students at 12 U.S. colleges and universities. Eating Behaviors, 2017; 24: 8I-88.

45. Dominguez-Whitehead Y. Food and housing challenges: (Re) framing exclusion in higher education. 2017. http://www.scielo.org.za/ scielo.php?script=sci_arttext\&pid=S2520-986820I 7000100009

46. Servilha E, Delatti M. College students' perception of classroom noise and its consequences on learning quality. Audiology - Communication Research, 2014; 19(2): 138-144.

https://doi: 10.1590/s23 17-64312014000200007

47. Joo S, Durband D, Grable J. The Academic Impact of Financial Stress on College Students. Journal of College Student Retention: Research, Theory \& Practice, 2008; 10(3): 287-305.

https://doi: $10.2190 / c s .10 .3 . c$

48. Lazarus RS, Folkman S. Stress, appraisal, and coping. New York: Springer; 1984.

49. Jan LK, Popescu L. Israel's nursing students' stress sources and coping strategies during their first clinical experience in hospital wards: A qualitative research. Revista de Asistenã Socialã, 2014; 13(4): 163-188.

50. Hoyne, G. \& McNaught, K., 20I3, 'Understanding the psychology of seeking support to increase Health Science student engagement in academic support services: A practice report', The International Journal of the First Year in Higher Education 4(I): 109-1 16. https://doi.org/10.5204/intjfyhe.v4il. 149

5I. King C, Edlington T, Williams B. The "Ideal" Clinical Supervision Environment in Nursing and Allied Health. Journal of Multidisciplinary Healthcare, 2020; 13: I87-196. https://doi:10.2147/jmdh.s239559

\section{Corresponding Author:}

*Robyn Henderson

Email address: robynhenderson I404@gmail.com 